\title{
Capillaria hepatica, A Case Report and Review of the Literatures
}

\author{
Maryam Kazemi Aghdam '; Abdollah Karimi ${ }^{2}$; Ali Amanati ${ }^{2,}$; Javad Ghoroubi ${ }^{3}$; Maliheh \\ Khoddami ${ }^{1}$; Bibi Shahin Shamsian ${ }^{4}$; Ahmad shamsizadeh ${ }^{5}$; Sara Zahedi Far ${ }^{6}$ \\ ${ }_{2}^{1}$ Pediatric Pathology Research Center, Mofid Children's Hospital, Shahid Beheshti University of Medical Sciences, Tehran, IR Iran \\ ${ }_{3}^{2}$ Pediatric Infections Research Center, Mofid Children's Hospital, Shahid Beheshti University of Medical Sciences, Tehran, IR Iran \\ 3 Pediatric Surgery Research Center, Mofid Children's Hospital, Shahid Beheshti University of Medical Sciences, Tehran, IR Iran \\ 4 Pediatric Congenital Hemathologic Disorders Research Center, Mofid Children's Hospital, Shahid Beheshti University of Medical Sciences, Tehran, IR Iran \\ 5 Infectious and Tropical Disease Research Center, Aboozar Children Hospital, Ahvaz Jundishapur University of Medical Sciences, Tehran, IR Iran \\ 6 Mofid Children's Hospital, Shahid Beheshti University of Medical Sciences, Tehran, IR Iran \\ ${ }^{*}$ Corresponding author: Ali Amanati, Pediatric Infections Research Center, Mofid Children's Hospital, Shahid Beheshti University of Medical Sciences, P. O. BOX:1546815514, Tehran, IR \\ Iran. Tel/Fax: +98-2122226941, E-mail:ali_amanati_1356@yahoo.com \\ Received: April 10, 2014; Revised: May 17, 2014; Accepted: June 6, 2014
}

\begin{abstract}
Introduction: Capillaria hepatica (Calodium hepaticum) is a parasite that cause very rare but life threatening infection in human beings. Case Presentation: The current paper reports a case of Capillaria hepatica infection in a four-year-old boy which presented with fever, hepatomegaly, peripheral eosinophilia, and noticeable weight loss. The diagnosis was made on the histological finding of degenerated nematode in the liver. He improved clinically by corticosteroid and albendazole therapy.

Conclusions: Capillaria hepatica should be considered in differential diagnoses in any child with fever, hepatomegaly, eosinophilia and hyperglobulinemia.
\end{abstract}

Keywords: Capillaria hepatica; Hepatic Granulomatous Reaction; Parasitic Infection

\section{Introduction}

Capillaria hepatica $(\mathrm{CH})$ is a worldwide zoonotic nematode, found in the liver of rodents including rats. Humans are rarely infected as the second host (1-5). The ova are present in the liver of primary host; soil gets contaminated by faces of the infected animal and it takes four to five weeks for the eggs to embryonate. The embryonated eggs are ingested by the second host through contaminated food, the larvae penetrate through intestinal wall to get into the portal venous system and maturate in the liver within 18 to 21 days. Pica is a risk factor, since it is more common in children, the infection is mostly detected in children between one to five years old (4-8). The adult form of parasite is thread-like and ova are similar to Trichuris trichiura ovum in appearance, but taper in its ends (9).

The most common symptoms are persistent fever, weight loss, hepatomegaly and peripheral eosinophilia. Hypergammaglobulinemia, high serum levels of alanine amino-transferase (ALT), aspartate amino-transferase (AST), and lactic dehydrogenase (LDH) are commonly observed $(4,6,9)$. Hepatic mass may be an unusual manifestation of the infection (2).

Imaging modalities such as ultrasound, CT scan and MRI are useful for diagnosis. Serologic methods like Indirect Immunoflorscent test and ELISA are also suggested; however, the presence of cross-reaction with echinococcus granulosus and echinococcus multilocularis is one of their limitations. Finally, liver biopsy and the presence of parasites ova and typical histology is the best method of diagnosis $(6,9,10)$.

Antihelminthic drugs (e.g. mebendazole, albendazole and thiabendazole) are used for therapy $(2,9,11)$; to control the fever and inflammation. Corticosteroids are also useful. In animal models, partial hepatectomy is suggested as an option to treat hepatic capillariosis $(3,9,12)$. Capillaria hepatica infection was not previously reported in Iran and the current case was the first reported.

\section{Case Presentation}

A four-year-old boy was admitted to the hospital with high-grade fever, chills, and weight loss (about $1.5 \mathrm{~kg}$ ) of two months duration. Hepatomegaly was noted in the physical examination. A liver ultrasound showed a relatively well-defined, but irregular isoechoic mass with a hypoechoic periphery measuring $58 \times 51 \times 47 \mathrm{~mm}$ in the VII segment of right lobe. An empirical one-week course of treatment with vancomycin and meropenem with preliminary diagnosis of liver abscess was not effective. Core needle biopsy of the liver mass suggested a parasitic infection; albendazole treatment had no clinical response.

He was referred to Mofid Pediatric Medical Center, Tehran, Iran, for further work-up. His complete blood count showed total white cell count $8400 / \mathrm{mm}^{3}$ with $8 \%$ eosinophil and mild anemia (Hb: $10.2 \mathrm{~g} / \mathrm{dL}$, normal 11-14 g/dL).

Copyright (C) 2015, Pediartric Infections Research Center. This is an open-access article distributed under the terms of the Creative Commons Attribution-NonCommercial 4.0 International License (http://creativecommons.org/licenses/by-nc/4.0/) which permits copy and redistribute the material just in noncommercial usages, provided the original work is properly cited. 
Kazemi Aghdam M et al.

Erythrocyte sedimentation rate was $97 \mathrm{~mm} /$ hour and Creactive protein level was 31. Liver enzymes were mildly elevated, AST: 220 IU/L; and ALT: 130 IU/L. Lumbar puncture and bone marrow aspiration were non-contributory. Serological tests results, including coombs wright, Strongiloid esstercolaris, and Toxocara antibodies, were negative. Ophthalmologic examination and echocardiography were also normal.

Abdominopelvic spiral computerized tomography (CT) scan without and with IV and oral contrast media revealed ill-defined mass lesion $61 \times 64 \times 65 \mathrm{~mm}$ with thick irregular peripheral enhancement and hypodense

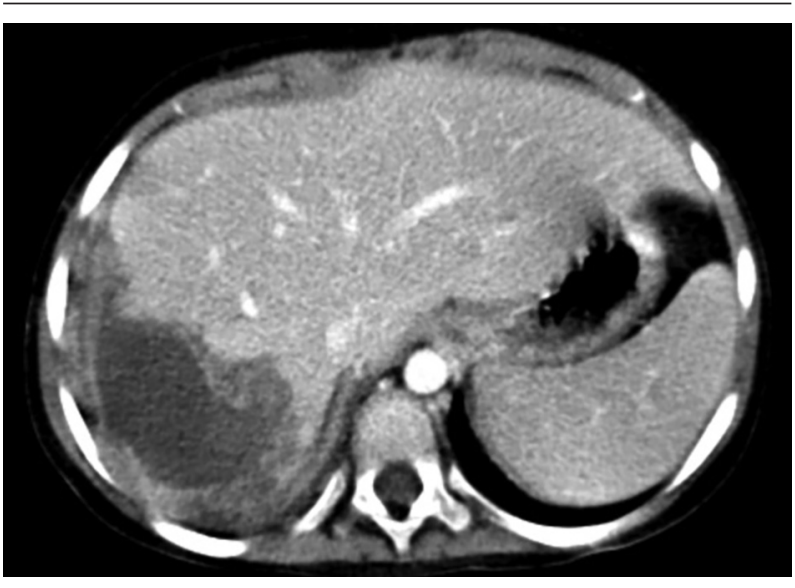

Figure 1. A Hypodense Lesion Measuring $65 \times 33 \mathrm{~cm}$ With Irregular Edematous Border in The Liver center in right lower liver lobe. The peripheral rim became relatively isodense with liver parenchyma in the delay phase (necrotic mass in favor of abscess) (Figure 1). Albendazole in combination with oral prednisolone was administrated and surgical excision was planned for abscess drainage.

Liver mass was reduced by surgical debulking. Specimen included the multiple irregular brown liver tissue fragments aggregating to $7 \times 5.3 \times 2 \mathrm{~cm}$, containing multiple well-defined tan nodules, ranging between 0.5 and $1.8 \mathrm{~cm}$ in diameter.

Microscopic examination showed that liver tissue was involved by extensive necrotizing granulomatous inflammation with severe eosinophilic infiltration (Figure $2 \mathrm{~A})$, many multi-nucleated foreign body type giant cells containing fragments of degenerating round worms (Figure 2B), as well as scattered degenerated worm body and unembryonated ova (Figures $2 \mathrm{C}$ and $\mathrm{D}$ ). These pathologic findings were the characteristics of Capillaria hepatica infestation. Diagnosis was made according to the pathologic findings along with the unique clinical presentation. Albendazole in combination with oral prednisolone was administrated after the diagnosis for more than six month. As a follow up program, he was regularly visited as an outpatient. Abdomen spiral CT scan without and with IV and oral contrast was done four weeks later. A hypodense lesion with irregular border with partial pripheral enhancement was observed segment VII of right middle lobe. The lesions showed considerable decrease in size compared with the primary study (Figure 3).

Figure 2. Microscopic Examination of Liver Biopsy With Haematoxylin and Eosin Stain $(\mathrm{H}$ and E, $\times 100)$
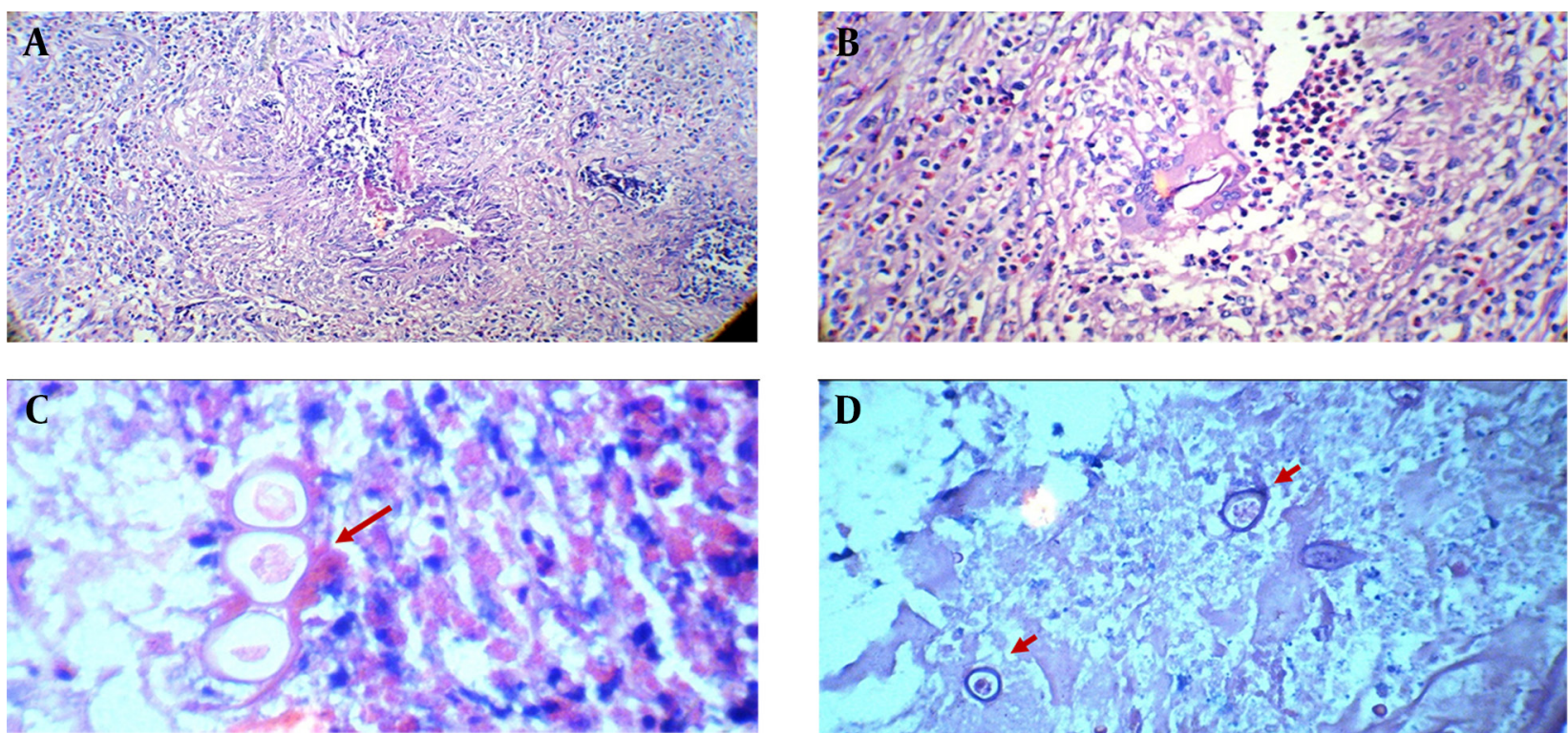

A, granulomatous inflammatory response with marked eosinophilic infiltration; B, multinucleated giant cell contains worm body with marked eosinophilic infiltration; C, degenerated worm body. D: Capillaria hepatica's ova in a massive necrotic background. 


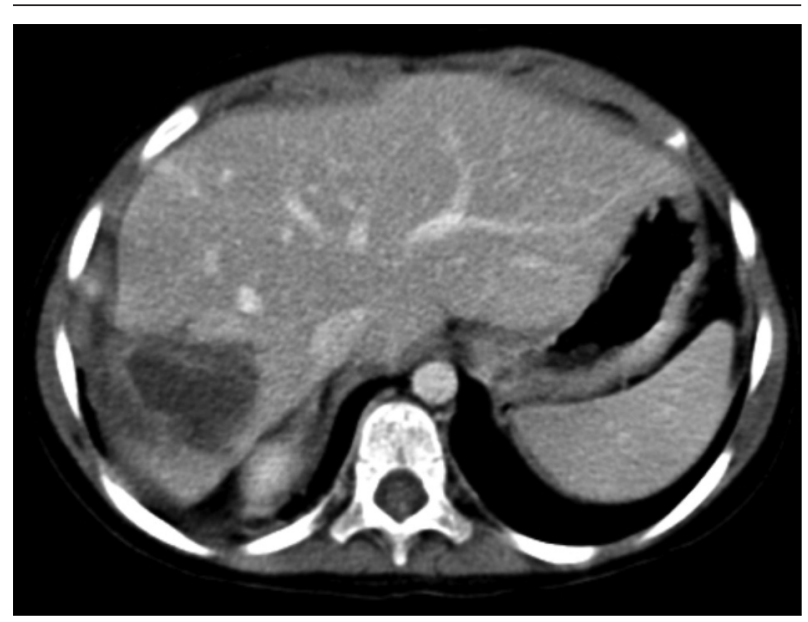

Figure 3. A Hypodense Lesion With Irregular Border With Partial Peripheral Enhancement Four Weeks After Treatment

\section{Discussion}

Capillaria hepatica (or Calodium hepaticum) is a nematode that globally infects the liver of mammals such as rodents, rabbits, dogs, and cats $(1,2,13)$. The first hosts are rodents, and rats are infected the most $(7,12)$. Capillaria hepatica is from superfamily Trichinelloidia $(3,7)$. Female worms lay eggs in mammalian liver where they mature. Inflammatory response destroys the adult worms. Eggs get released after the hosts' death. Once the secondary host uses the infected water or food, the ingested eggs develop to larvae, which pass through intestinal wall and migrate through portal system to liver where they turn into adult worms in a period of four weeks $(6,13)$. This infection is reported in Japan, India, America, Canada, Brazil, and temperate and tropical zones $(1,9,12)$. Females are infected more than males and most of the patients are from a very low socioeconomic class. Until 2012, fewer than 100 cases of Capillaria hepatica infestation was reported in humans (1, 3). If human ingests unembryonated eggs and the eggs pass the gastrointestinal lumen without any inflammatory reaction or host response, the condition is a spurious infection and is diagnosed only by stool exam. This type of infection is mostly asymptomatic but may present with mild diarrhea. Capillaria hepatica mostly occurs in children under the age of three years; however, it can also happen in adults (reported age range: 14 months to 78 years) (3). This disorder manifests non-specific symptoms such as persistent fever, hepatomegaly, eosinophilia and sometimes leads to death. The current case was a four-year-old boy with high-grade fever at presentation. This nematode causes granulomatous reaction and fibrosis in the liver. In early stages, there are immature worms without eggs but in advanced cases, there are adult worms with eggs in the liver. If the patient survives, in long lasting cases, adult worms vanish and fibrosis and local inflammation remains in the liver (9).

Since $C$. hepatica can be lethal, it should be considered in differential diagnosis in a child with fever, hepatomegaly, eosinophilia and hyperglobulinemia associated with increased AST, ALT and LDH levels (6). Capillaria hepatica is considered as a significant etiology of eospinophilic granuloma of the liver, although Toxocara canis is the most common cause (2). The current case, diagnosed by histological examination of the resected liver mass and was treated successfully, was the first case reported from Iran.

\section{Acknowledgements}

The authors would like to thank the Pediatric Pathology Research Center and Pediatric Infection Research Center staff of Mofid Children's Hospital.

\section{Authors' Contributions}

All the authors equaly contributed in writing this article.

\section{References}

1. Wang Z, Lin X, Wang Y, Cui J. The emerging but neglected hepatic capillariasis in China. Asian Pac J Trop Biomed. 2013;3(2):146-7.

2. Klenzak J, Mattia A, Valenti A, Goldberg J. Hepatic capillariasis in Maine presenting as a hepatic mass. Am J Trop Med Hyg. 2005;72(5):651-3.

3. Fuehrer HP, Igel P, Auer H. Capillaria hepatica in man--an overview of hepatic capillariosis and spurious infections. Parasitol Res. 2011;109(4):969-79.

4. Lucas SB. Other viral and infectious diseases and HIV-related liver disease. Pathology of the liver. 4 ed: Churchill Livingstone; 2002.

5. Nabi F, Palaha HK, Sekhsaria D, Chiatale A. Capillaria hepatica infestation. Indian Pediatr. 2007;44(10):781-2.

6. Silverman NH, Katz JS, Levin SE. Capillaria hepatica infestation in a child. S Afr Med J.1973;47(6):219-21.

7. health PA. Capillariasis Centers for Disease Control and Prevention. 2009. Available from: http://www.dpd.cdc.gov/dpdx/HTML/Capillariasis.htm.

8. Wongsawasdi L, Ukarapol N, Lertprasertsuk N. The endoscopic diagnosis of intestinal capillariasis in a child: a case report. Southeast Asian J Trop Med Public Health. 2002;33(4):730-2.

9. Tesana S, Puapairoj A, Saeseow O. Granulomatous, hepatolithiasis and hepatomegaly caused by Capillaria hepatica infection: first case report of Thailand. Southeast Asian J Trop Med Public Health. 2007;38(4):636-40.

10. Juncker-Voss M, Prosl H, Lussy H, Enzenberg U, Auer H, Nowotny N. Serological detection of Capillaria hepatica by indirect immunofluorescence assay. J Clin Microbiol. 2000;38(1):431-3.

11. Sawamura R, Fernandes MI, Peres LC, Galvao LC, Goldani HA, Jorge SM, et al. Hepatic capillariasis in children: report of 3 cases in Brazil. Am J Trop Med Hyg. 1999;61(4):642-7.

12. Li CD, Yang HL, Wang Y. Capillaria hepatica in China. World J Gastroenterol. 2010;16(6):698-702.

13. Terrier P, Hack I, Hatz C, Theintz G, Roulet M. Hepatic capillariasis in a 2-year-old boy. J Pediatr Gastroenterol Nutr. 1999;28(3):338-40. 